\title{
Upstaging nodal status in colorectal cancer using ex vivo fluorescence sentinel lymph node mapping: preliminary results
}

\author{
Andrea Picchetto, Michele Diana , Lee L. Swanström , Fabio Massimo \\ Magliocca , Annamaria Pronio, Eleonore Choppin , Stefania La Rocca, \\ Jacques Marescaux \& Giancarlo D'Ambrosio
}

To cite this article: Andrea Picchetto , Michele Diana , Lee L. Swanström , Fabio Massimo Magliocca , Annamaria Pronio , Eleonore Choppin , Stefania La Rocca , Jacques Marescaux \& Giancarlo D'Ambrosio (2020): Upstaging nodal status in colorectal cancer using ex vivo fluorescence sentinel lymph node mapping: preliminary results, Minimally Invasive Therapy \& Allied Technologies, DOI: 10.1080/13645706.2020.1798464

To link to this article: https://doi.org/10.1080/13645706.2020.1798464

\section{曲 Published online: 31 Jul 2020.}

Submit your article to this journal 준

\section{Џ Article views: 5}

Q View related articles $\square$

View Crossmark data $\longleftarrow$ 


\title{
Upstaging nodal status in colorectal cancer using ex vivo fluorescence sentinel lymph node mapping: preliminary results
}

\author{
Andrea Picchetto $^{\mathrm{a}}$ (D), Michele Diana ${ }^{\mathrm{b}, \mathrm{c}}$, Lee L. Swanström ${ }^{\mathrm{b}}$, Fabio Massimo Magliocca ${ }^{\mathrm{d}}$, Annamaria \\ Pronio $^{a}$, Eleonore Choppin ${ }^{a}$, Stefania La Rocca ${ }^{a}$, Jacques Marescaux ${ }^{b, c}$ and Giancarlo D'Ambrosio ${ }^{a}$ \\ ${ }^{a}$ General and Colorectal Surgery Division, Department of Cardiothoracic, Vascular Surgery and Organ Transplantation, Policlinico

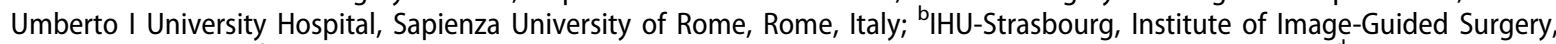 \\ Strasbourg, France; 'IRCAD, Research Institute against Cancer of the Digestive System, Strasbourg, France; ${ }^{\mathrm{d}}$ Department of \\ Radiological, Oncological and Pathological Sciences, Sapienza University of Rome, Policlinico Umberto I University Hospital, \\ Rome, Italy
}

\begin{abstract}
Background: Sentinel lymph node (SLN) mapping using near-infrared fluorescence (NIRF) imaging is a recent technique to improve nodal staging in several tumors. The presence of colorectal cancer (CRC) micro-metastases has recently been defined as N1 disease and no longer as N1 mi, determining the need for adjuvant chemotherapy. In CRC, the reported rate of SLN micro-metastases detected by ultrastaging techniques is as high as $30 \%$. The aim of this prospective study is to report the preliminary results of the sensitivity analysis of NIRF imaging for ex vivo SLN mapping and the research of micro-metastases in CRC, in patients with node-negative disease (NND).

Material and methods: On the specimen of 22 CRC patients, $1 \mathrm{~mL}$ of ICG $(5 \mathrm{mg} / \mathrm{mL})$ was injected submucosally around the tumor to identify SLNs. NND SLNs were further investigated with ultrastaging techniques.

Results: Three-hundred and sixty-three lymph nodes were retrieved (59 SLNs; mean per case: 2.7). The detection, sensitivity and false-negative rate were $100 \%, 100 \%$ and $0 \%$ respectively. Ultrastaging investigations showed no micro-metastases in the NND SLNs.

Conclusions: The ex vivo SLN fluorescence-based detection in CRC was confirmed to be easy to perform and reliable. In this preliminary results report of an ongoing study, the SLN assay was congruent with the nodal status, as confirmed by histological investigations.
\end{abstract}

\section{ARTICLE HISTORY}

Received 18 January 2020

Accepted 26 June 2020

\section{KEYWORDS}

Near-infrared fluorescence imaging; fluorescenceguided surgery; sentinel lymph node mapping; colorectal cancer; ultrastaging

\section{Introduction}

Colorectal cancer (CRC) is the second most common cancer, and the second most common cause of cancer-related deaths in Europe [1].

Nodal staging is a crucial prognostic factor, which determines the need for adjuvant chemotherapy [2]. Patients presenting with a node-negative disease (NND) may experience a five-year overall survival (OS) in $70-80 \%$ of cases, and this decreases to $30-60 \%$ for patients presenting with positive nodes $(\mathrm{N}+)$. Occult lymph node metastases could be responsible for approximately 20-30\% of disease recurrences occurring in (apparently) NND patients [3].

The current recommendation, despite the lack of sufficient evidence, is to obtain a minimum of 12 lymph nodes in the resection specimen $[3,4]$.
According to the last edition (8th) of the AJCC Cancer Staging Manual (January 2018), the presence of lymph node micro-metastases should be staged as $\mathrm{N} 1$ and no longer as N1mi (as it was done in the 7th edition) $[5,6]$. This is a major change and influences patient treatment as there is strong evidence that adjuvant chemotherapy improves $\mathrm{OS}$ in $\mathrm{N}+$ cases $[3,7]$. Colorectal micro-metastases are not typically seen on pre-operative imaging and are frequently missed on standard pathologic survey. They are best detected by ultrastaging techniques including serial sectioning and additional immunohistochemistry.

Sentinel lymph nodes (SLNs) are defined as the first lymph nodes in the lymphatic pathway draining a primary solid malignancy [8]. The rationale behind the intraoperative identification and analysis of the SLNs lies in their status, which can predictively

CONTACT Andrea Picchetto andrea.picchetto@uniroma1.it General and Colorectal Surgery Division, Department of Cardiothoracic, Vascular Surgery and Organ Transplantation, Policlinico Umberto I University Hospital, Viale del Policlinico 155, Rome, Italy

This work was presented at the 26th Conference of the European Association for Endoscopic Surgery (EAES), in London, United Kingdom (30 May-1 June 2018).

(C) 2020 Society of Medical Innovation and Technology 
describe the downstream nodal basin. This concept and method for SLN assessment is highly standardized in several cancers, including melanoma, breast or head and neck cancers. However, when applied to colorectal cancer, the concept of SLN is more controversial, since it does not typically influence the surgical strategy. Nevertheless, intraoperative SLN identification could lead to an ultrastaging strategy whereby sentinel nodes could be subjected to additional testing to detect micro-metastases, potentially upstaging patients and influencing their prognosis and treatment strategies [9]. Additionally, the concept of SLN could become more relevant with the increasing number of organ-sparing, localized procedures, such as endoscopic submucosal dissections (ESDs) or limited full-thickness resections (FTR), which can be considered oncologically safe only if lymph nodes are not involved [10].

SLN mapping is currently performed via a peritumoral injection of a radioactive tracer, or a visible blue dye such as isosulfan blue (IB), or with a combination of radiotracer and vital dye in a dual modality technique. However, in colorectal cancer, the concept and methods for SLN navigation and mapping lack evidence of both clinical relevance and standardization [11-13].

SLN mapping in CRC was initially suggested by Saha et al. during the 50th annual cancer symposium of the Society of Surgical Oncology in 1997. It has since been described by various authors as having favorable but inconstant outcomes and accuracy rates, mainly due to the diversity of the mapping methods which were scrutinized [14-18].

Blue dyes and radiotracers have been used as SLN tracers in both in vivo and ex vivo settings. However, both have some disadvantages, that is, vital dyes can be difficult to visualize through adipose tissue, while gamma ray-emitting radiotracers expose patients and caregivers to ionizing radiation, require the involvement of a nuclear medicine facility and entail high management costs $[9,11,19]$.

A promising alternative to current techniques of real-time lymph node mapping is provided by the emerging concept of fluorescence-guided surgery $[20,21]$. Upon a peritumoral injection of a fluorophore (i.e. a substance emitting a fluorescence signal after being illuminated by a near-infrared light source), it is possible to highlight both the lymphatic pathway and the primary draining lymph nodes [22].

Near-infrared lymphography by means of peritumoral injection of Indocyanine Green (ICG) has some additional advantages over any blue dye method since the light at the NIR wavelength provides a deeper tissue penetration.

The ICG concentration reported in various trials varies from $2.5 \mathrm{mg} / \mathrm{mL}$ to $5 \mathrm{mg} / \mathrm{mL}$ and most authors apply 1 to $4 \mathrm{ml}$ per neoplastic site, generally three to five minutes prior to visualization [23]. The authors chose to use the ex-vivo submucosal injection in order not to inject the ICG in the patient (thus avoiding drug reaction) and to easily manage the specimen in order to reduce the ICG's spillage.

The aim of this preliminary report of an ongoing prospective study is to analyze the predictability, intended as detection, sensitivity and false-negative rate, of NIR fluorescence imaging for ex vivo SLN research in conventional surgical resection for colorectal tumors. Secondary endpoint is to assess the prevalence of micro-metastases in patients with nodenegative disease (NND).

\section{Material and methods}

\section{Patients}

Between January 2017 and March 2018, 22 patients presenting with resectable CRC and without distant metastases at imaging (iTxNxM0) were selected and enrolled in this ongoing prospective study.

The study received approval by the Ethical Committee of the University Hospital Policlinic Umberto I, 'Sapienza' University of Rome, Italy. All patients accepted and signed an informed consent form.

The preliminary evaluation of the first 22 patients was required to confirm the feasibility of the treatment before completing the enrolment of additional 44 patients and involving other surgical divisions.

Overall patient demographics and tumor characteristics are reported in Table 1.

All patients (seven) with rectal cancer presented a locally advanced stage and underwent standard longcourse neoadjuvant radio-chemotherapy [24,25]. These patients were included to better understand if this technique should be a useful tool to recognize the SLNs even in the irradiated mesorectum.

The number of SLNs per patient, the detection rate (DR), the sensitivity, the false-negative (FN) rate, and the upstaging rate were calculated.

The detection rate was calculated as the number of patients (with SLNs identified by means of ICG-NIR fluorescence) $\times 100$, divided by the number of all enrolled patients.

The sensitivity rate of SLN mapping was defined as the number of patients with a positive SLN $\times 100$, 
Table 1. Patient characteristics.

\begin{tabular}{lcc}
\hline & All pts $(n=22)$ & NND pts (n10) \\
\hline Age (ys) median and range & $73,2(59-88)$ & $69(53-81)$ \\
BMI (kg/m²), mean & $24.57(19.8-29.2)$ & $24.82(20.1-29.8)$ \\
Neoplasia localization & & \\
Right colon & $5(22.7 \%)$ & $2(20 \%)$ \\
Splenic flexure & $1(4.5 \%)$ & $1(10 \%)$ \\
Left colon & $3(13.6 \%)$ & $1(10 \%)$ \\
Sigmoidorectal junction & $6(27.3 \%)$ & $3(30 \%)$ \\
Rectum & $7(31.8 \%)$ & $3(30 \%)$ \\
Procedure & & $2(20 \%)$ \\
Right colectomy & $5(22.7 \%)$ & $1(10 \%)$ \\
Atypical colectomy & $1(4.5 \%)$ & $1(10 \%)$ \\
Left colectomy & $3(13.6 \%)$ & $6(60 \%)$ \\
RAR & $13(59.1 \%)$ & $3(30 \%)$ \\
Tumor staging by histology & & $2(20 \%)$ \\
pT1 & $5(22.7 \%)$ & $4(40 \%)$ \\
pT2 & $5(22.7 \%)$ & $1(10 \%)$ \\
pT3 & $9(40.9 \%)$ & not applicable \\
pT4 & $3(13.6 \%)$ & \\
Nodal staging & & \\
pN0 & $10(45.5 \%)$ & \\
pN1 & $5(22.7 \%)$ & $16.4(164)$ \\
pN2 & $6(27.3 \%)$ & $2.5(1-6)$ \\
pN3 & $1(4.5 \%)$ & $0 / 10(0 \%)$ \\
Mean number (total) of LN excised & $16,5(363)$ & \\
Mean number (range) of SLN excised & $2,7(1-9)$ & \\
Micrometastases & not applicable &
\end{tabular}

divided by the number of patients with any positive lymph node after dissection and histopathological preparation of the lymph node basin.

False negatives represented the proportion of patients with SLNs without apparent tumor cells but tumor-positive in non-SLNs.

The upstaging rate was assumed as the proportion of patients with a micrometastatic disease, with negative nodes found by means of conventional pathological examination, by performing a ultrastaging examination of the SLNs.

\section{Procedures}

All patients underwent standard oncological laparoscopic resections, as follows: right colectomies $(n=5)$, left colectomies $(n=3)$, rectal anterior resections $(n=13)$, atypical colectomy $(n=1)$.

For the NIRF detection, the Quest Spectrum (Quest Medical Imaging, Middenmeer, the Netherlands), the Stryker (Stryker, Kalamazoo, MI, USA) and the Storz Imaging Systems (Karl Storz, Tuttlingen, Germany) were used.

In order to standardize the NIRF SLN research, we performed the ex vivo technique as previously described in the literature. The intact surgical specimen was opened longitudinally at the back table in the OR and $1 \mathrm{ml}$ of indocyanine green (ICG, $5 \mathrm{mg}$ / $\mathrm{mL}$ ) was injected submucosally at four corners (Figure 1) around the tumor in order to identify the lymphatic pathway and the SLNs.

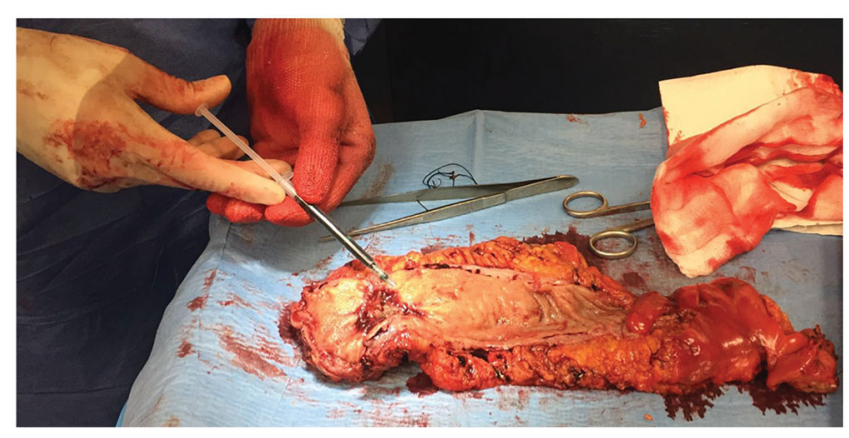

Figure 1. Ex vivo ICG submucosal injection.

After a mean latency of seven to 10 minutes, it was possible to identify both the lymphatic channels (Figure 2) and the receiving nodes by means of fluorescence imaging.

As reported by several authors, the first one to four nodes that became fluorescent in the first $15 \mathrm{~min}$ were identified as SLNs, were dissected free from the mesocolon or mesorectum (Figure 3), and were labelled and sent to the pathologist $[9,18,26]$. The remaining intact specimen was further investigated as usual (without NIRF) by the pathologist to retrieve the other lymph nodes and perform the anatomopathological staging.

\section{Histopathology}

All specimens were investigated using conventional pathology techniques. SLNs were grossly sectioned at 


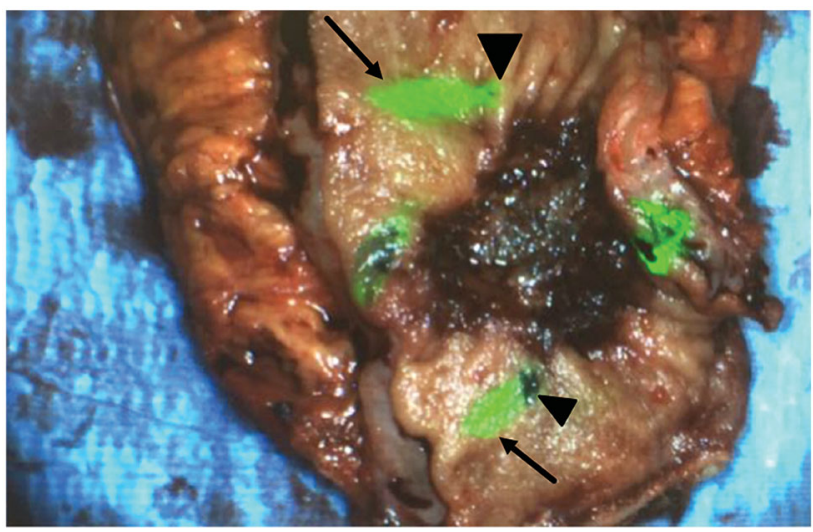

Figure 2. NIRF lymphatic channels. Arrowhead: Injection site. Arrows: lymphatic channel.

$2 \mathrm{~mm}$ intervals and each section was sent for pathological analysis. The SLNs were analyzed according to current oncological guidelines by two experienced pathologists in a blinded fashion.

Each SLN in patients who presented with NND after performing a conventional histological analysis was further investigated with ultrastaging techniques in order to detect the presence of micro-metastases (defined as clusters of 10 to 20 tumor cells or clumps of tumor cells $\geq 0.2 \mathrm{~mm}$ in diameter), including serial sectioning and additional immunohistochemistry. In the 8th edition of the AJCC Cancer Staging Manual and Handbook, micro-metastases were defined as standard positive nodes (N1) and no longer as micrometastases $(\mathrm{N} 1 \mathrm{mi})$ as they were in the 7 th edition $[2,5]$.

Ultrastaging of the SLNs was performed at five multilevel micro-sections of $30 \mu \mathrm{m}$, with four sections stained with $\mathrm{H} \& \mathrm{E}$ at $4 \mathrm{X}$ and $10 \mathrm{X}$ magnification and one immuno-stained for cytokeratin AE-1/AE3 cocktail according to the Novocastra protocol (Bond Leica Systems) at $4 \mathrm{X}$ and $10 \mathrm{X}$ magnification. The remainder of the specimen, including all non-SLNs, was examined using standard pathological methods. All non-SLNs were retrospectively ultrastaged by performing five additional sections for $\mathrm{H}$ and $\mathrm{E}$ at $30 \mu \mathrm{m}$.

\section{Results}

For the conventional pathology, the $\mathrm{pT}$ stages in 22 patients were distributed as follows: pT1 $(n=5), \mathrm{pT} 2$ $(n=5), \quad$ pT3 $\quad(n=9), \quad$ and $\quad$ pT4 $\quad(n=3)$ adenocarcinomas.

A total of 363 nodes were retrieved (mean: 16.5; range 4-32). The total number of SLNs was 59 (mean: 2.7, range: 1-9). The pathological nodal staging was as follows: $10 \mathrm{pN} 0$, five $\mathrm{pN} 1$, six $\mathrm{pN} 2$ and one $\mathrm{pN} 3$, for a total of $12 \mathrm{~N}+$ (Table 1 ).

The detection rate was $100 \%(22 / 22 \mathrm{pts})$.

The conventional histopathological investigations showed that every patient with metastatic SLN had one or more metastatic non-SLN thus reporting a $100 \%$ sensitivity rate $(12 \mathrm{SLN}+/ 12 \mathrm{~N}+$ pts) (Table 2$)$.

The 10 patients with node-negative disease (pN0) were further investigated and their characteristics are summed up in Table 1.

There were no perineural invasions in any NND patients and lymphovascular invasion was reported in four cases.

The total number of retrieved nodes was 164 (mean: 16.4, range: 4-32, standard deviation \pm 7.95 ). At least one SLN was found in all cases (mean: 2.5, range: $1-6$, total: 25 ).

There were no false-negative cases (0/10 NND pts).

The ultrastaging histopathological investigations did not show any isolated tumor cells or micro-metastases in the SLNs, with consequently a O\% upstaging rate.

SLN located deeper in the mesocolic and mesorectal fat could be easily identified by means of NIRF with ICG, even in the irradiated mesorectum (Figure 3).

\section{Discussion}

The detection of micro-metastases in CRC patients has recently been adopted as an element in the identification of patients who are candidates for adjuvant chemotherapy.

Intraoperative near-infrared fluorescence imaging with the use of ICG as a fluorophore is an increasingly performed technique: for organ perfusion, anatomy identification and also for mapping of lymphatic drainage $[27,28]$. NIRF has been shown to allow SLN detection in colorectal specimens in both in vivo and ex vivo settings. Several authors have reported on colorectal SLN research using vital blue dyes and/or radiotracers. However, results were not compelling as methods were not standardized, especially in patients presenting with rectal cancer and operated after nChRT and clinical care pathways were not affected $[11,13]$. One problem with past attempts to validate the use of sentinel node has been the absence of an arm that looks at ultrastaging or determination of micro metastases using immunohistopathologic techniques.

Chand et al. reported a preliminary series of 10 patients (FLICC study) with a relatively low detection 


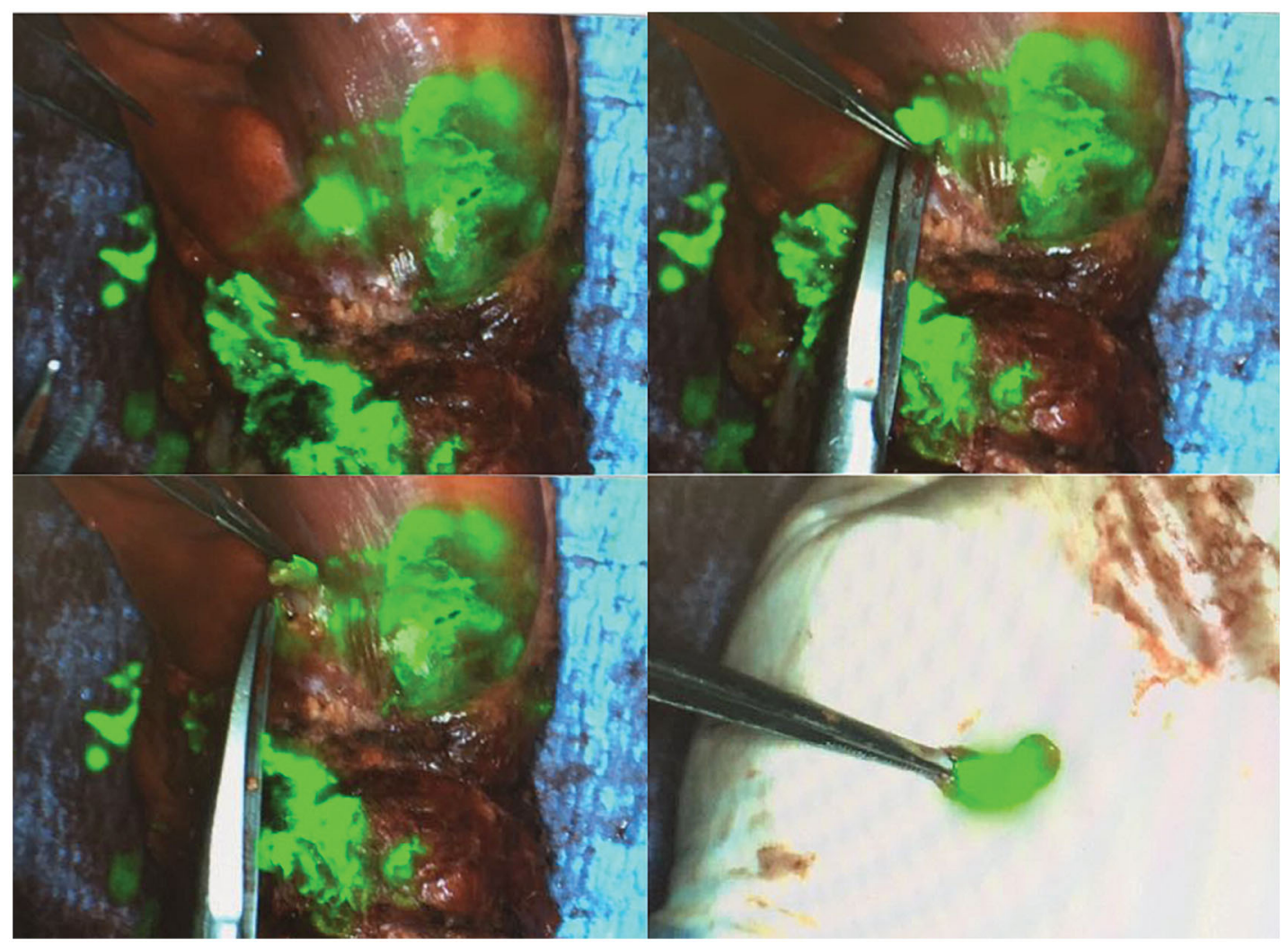

Figure 3. SLN identification and dissection.

Table 2. Results.

\begin{tabular}{ll}
\hline Detection rate & $100 \%(22 / 22 \mathrm{pts})$ \\
Sensitivity rate & $100 \%(12 \mathrm{SLN}+/ 12 \mathrm{~N}+\mathrm{pts})$ \\
False negative rate & $0 \%(0 / 10 \mathrm{pts})$ \\
Upstaging rate & $0 \%(0 / 10 \mathrm{NND}$ pts $)$ \\
\hline
\end{tabular}

rate $(80 \%)$ but using no ultrastaging investigations [29].

Concerning the potential impact of ultrastaging, Wiese et al. reported a significantly higher rate of upstaging when applying ultrastaging methods to SLNs (12\%), compared to non-SLNs (only $1 \%$ of upstaging) in a cohort of 200 patients with CRC (presenting with a preoperative node-negative disease) [8].

Rivet reported a detection rate of $98 \% \quad(56 / 57$ patients), a sensitivity of $48 \%$, and an $\mathrm{FN}$ rate of $52 \%$ with an upstaging rate of $5.26 \%(3 / 57)$ of patients with NND after SLN mapping with isosulfan blue (IB) [30].

Park, when comparing the ex vivo and in vivo injection of blue dye in sentinel lymph node mapping for colorectal cancer, reported similar though slightly higher detection rates (for the ex vivo technique) (90.6 vs. $81.1 \%)$, sensitivity ( 86.7 vs. $76.5 \%), \mathrm{FN}(13.3$ vs. $23.5 \%)$ and upstaging rates (21.4 vs. $15.3 \%)$ (overall US rate: $18.5 \%, 5 / 27$ patients) [31].
Andersen et al. compared the in vivo (with ICG) and ex vivo (with methylene blue) SLN mapping in the same patients. In a series of 29 patients, the authors reported a combined detection rate of $75.9 \%$. In seven of the enrolled patients, no SLN could be detected using either technique. When considered separately, the SLN detection rate dropped to $65.6 \%$ for in vivo and to $37.9 \%$ for ex vivo analysis. Additionally, no micro-metastases were found. They used a different recognition timing, both for the in vivo (20 min) and the ex vivo (two minutes) technique. This could probably account for their lower detection rate when compared to other series [32].

Weixler et al. compared the in vivo (with isosulfan blue, $n=170$ ) versus the ex vivo (with HSA800 IRDye800CW combined with human serum albumin, $n=50)$ SLN mapping in a series of 220 stage I-III patients. A similar detection rate was achieved in both the isosulfan group (100\%) and the HSA800 group (98\%). The sensitivity, $\mathrm{FN}$ and US rates were 75.3 versus $64 \%, 24.7$ versus $36 \%$, and 25.8 versus $17.2 \%$ respectively [33].

Hirche et al. applied the NIR ICG in vivo technique in a series of 26 patients and achieved a 96\% detection rate with an $82 \%$ sensitivity and an $18 \%$ FN. Upstaging occurred in $16 \%$ of NND patients (3/ 18) $[23]$. 
About the SLN research in patients with rectal cancer who had undergone neoadjuvant radio-chemotherapy, several authors have reported their series using different tracers (i.e. radiotracers, blue dyes, etc.).

Braat et al. applied an ex-vivo technique with Patent Blue V, in a series of 34 patients who had undergone neoadjuvant treatment, identifying the SLN in 26 of 34 patients [26].

Lezoche et al. used the $99 \mathrm{~m}$-technetium-marked nanocolloid during endoluminal locoregional resection (ELRR) by transanal endoscopic microsurgery (TEM) reporting a detection rate of $61,5 \% \quad(8 / 13$ pts) [34].

Similarly, Arezzo et al. performed a transrectal sentinel lymph node biopsy for early rectal cancer during transanal endoscopic microsurgery using the NIR ICG reporting a $100 \%$ detection rate on three patients [35].

Our results are similar to those and confirm the validity of this methodology.

In our series, a total of 59 SLNs (mean: 2.7) were detected $(100 \%)$ with a $100 \%$ sensitivity and no FN proving the efficacy of benchtop SNL identification. Each NND SLN at conventional histological analysis was further investigated with ultrastaging techniques. No micro-metastases were found in those SLNs, and as a result, none of our patients were upstaged. The absence of micro-metastases in the investigated SLN may be due to the small sample size, and more cases should be collected to corroborate this finding.

\section{Conclusions}

Ex vivo SLN fluorescence-based detection in colorectal cancer was confirmed to be easy-to-perform and reliable. In this preliminary series, sentinel lymph node assay was concordant with the locoregional nodes status, as confirmed by histological investigations and may provide, at least in ex vivo setting, a resource sparing practice algorithm for ultrastaging based on micrometastasis detection.

\section{Declaration of interest}

Andrea Picchetto, Lee L. Swanstrom, Fabio Massimo Magliocca, Annamaria Pronio, Eleonore Choppin, Stefania La Rocca, and Giancarlo D'Ambrosio have no conflicts of interest or financial ties to disclose. Michele Diana is the recipient of a grant from the French Foundation ARC.

\section{ORCID}

Andrea Picchetto (D) http://orcid.org/0000-0003-4300-9286

\section{References}

[1] Ferlay J, Soerjomataram I, Dikshit R, et al. Cancer incidence and mortality worldwide: sources, methods and major patterns in GLOBOCAN 2012. Int J Cancer. 2015;136(5):E359-386.

[2] Benson AB, Venook AP, Al-Hawary MM, et al. NCCN guidelines insights: colon cancer, version 2.2018. J Natl Compr Canc Netw. 2018;16(4): 359-369.

[3] Ong MLH, Schofield JB. Assessment of lymph node involvement in colorectal cancer. World J Gastrointest Surg. 2016;8(3):179-192.

[4] Le Voyer TE, Sigurdson ER, Hanlon AL, et al. Colon cancer survival is associated with increasing number of lymph nodes analyzed: a secondary survey of intergroup trial INT-0089. J Clin Oncol. 2003; 21(15):2912-2919.

[5] Amin MB. Edge SB. American joint committee on cancer. AJCC cancer staging manual. 4th ed Switzerland: Springer; 2017.

[6] Edge SB. American Joint Committee on Cancer. AJCC cancer staging manual. 7th ed. New York: Springer; 2010.

[7] Fang SH, Efron JE, Berho ME, et al. Dilemma of stage II colon cancer and decision making for adjuvant chemotherapy. J Am Coll Surg. 2014;219(5): 1056-1069.

[8] Wiese D, Sirop S, Yestrepsky B, et al. Ultrastaging of sentinel lymph nodes (SLNs) vs. non-SLNs in colorectal cancer-do we need both?. Am J Surg. 2010; 199(3):354-358.

[9] van der Pas MH, Meijer S, Hoekstra OS, et al. Sentinel-lymph-node procedure in colon and rectal cancer: a systematic review and meta-analysis. Lancet Oncol. 2011;12(6):540-550.

[10] Cahill RA, Leroy J, Marescaux J. Localized resection for colon cancer. Surg Oncol. 2009;18(4):334-342.

[11] Vahrmeijer AL, Hutteman M, van der Vorst JR, et al. Image-guided cancer surgery using near-infrared fluorescence. Nat Rev Clin Oncol. 2013;10(9): 507-518.

[12] Hutteman M, Choi HS, Mieog JS, et al. Clinical translation of ex vivo sentinel lymph node mapping for colorectal cancer using invisible near-infrared fluorescence light. Ann Surg Oncol. 2011;18(4): 1006-1014.

[13] Zhu B, Sevick-Muraca EM. A review of performance of near-infrared fluorescence imaging devices used in clinical studies. Br J Radiol. 2015;88(1045): 20140547.

[14] Saha S, Wiese D, Badin J, et al. Technical details of sentinel lymph node mapping in colorectal cancer and its impact on staging. Ann Surg Oncol. 2000; $7(2): 120-124$.

[15] Saha S, Bilchik A, Wiese D, et al. Ultrastaging of colorectal cancer by sentinel lymph node mapping 
technique-a multicenter trial. Ann Surg Oncol. 2001;8(9 Suppl):94S-98S.

[16] Wood TF, Saha S, Morton DL, et al. Validation of lymphatic mapping in colorectal cancer: in vivo, ex vivo, and laparoscopic techniques. Ann Surg Oncol. 2001;8(2):150-157.

[17] Kitagawa Y, Fujii H, Mukai M, et al. The role of the sentinel lymph node in gastrointestinal cancer. Surg Clin North Am. 2000;80(6):1799-1809.

[18] Picchetto A, Seeliger B, La Rocca S, et al. Fluorescence-guided detection of lymph node metastases of gastrointestinal tumors. Chirurg. 2019; 90(11):891-898.

[19] Schaafsma BE, Verbeek FP, van der Vorst JR, et al. Ex vivo sentinel node mapping in colon cancer combining blue dye staining and fluorescence imaging. J Surg Res. 2013;183(1):253-257.

[20] Diana M. Enabling precision digestive surgery with fluorescence imaging. Transl Gastroenterol Hepatol. 2017;2:97.

[21] Diana M. Fluorescence-guided surgery applied to the digestive system: the cybernetic eye to see the invisible. Cir Esp. 2018;96(2):65-68.

[22] Nishigori N, Koyama F, Nakagawa $\mathrm{T}$, et al. Visualization of lymph/blood flow in laparoscopic colorectal cancer surgery by ICG fluorescence imaging (Lap-IGFI). Ann Surg Oncol. 2016;23(Suppl 2): S266-S274.

[23] Hirche C, Mohr Z, Kneif S, et al. Ultrastaging of colon cancer by sentinel node biopsy using fluorescence navigation with indocyanine green. Int J Colorectal Dis. 2012;27(3):319-324.

[24] Benson AB, Venook AP, Al-Hawary MM, et al. Rectal Cancer, Version 2.2018, NCCN Clinical Practice Guidelines in Oncology. J Natl Compr Canc Netw. 2018;16(7):874-901.

[25] Glynne-Jones R, Wyrwicz L, Tiret E, et al.; ESMO Guidelines Committee. Rectal cancer: ESMO Clinical Practice Guidelines for diagnosis, treatment and follow-up. Ann Oncol. 2017;28(suppl_4):iv22-iv40.

[26] Braat AE, Oosterhuis JW, Moll FC, et al. Sentinel node detection after preoperative short-course radiotherapy in rectal carcinoma is not reliable. Br J Surg. 2005;92(12):1533-1538.
[27] Heiliger C, Piecuch J, Frank A, et al. Intraarterial indocyanine green (ICG) fluorescence augmentation by marking embryonal resection areas in colorectal surgery: a feasibility study in a porcine model. Minim Invasive Ther Allied Technol. 2019;28(6): 321-325.

[28] Frank AHR, Heiliger C, Andrade D, et al. Intraarterial versus negative-staining of embryonal resection borders with indocyanine green (ICG) fluorescence for total mesorectal excision in colorectal cancer - an experimental feasibility study in a porcine model. Minim Invasive Ther Allied Technol. 2020 [e-pub ahead of print May 19, 2020].

[29] Chand M, Keller DS, Joshi HM, et al. Feasibility of fluorescence lymph node imaging in colon cancer: FLICC. Tech Coloproctol. 2018;22(4):271-277.

[30] Rivet EB, Mutch MG, Ritter JH, et al. Ex vivo sentinel lymph node mapping in laparoscopic resection of colon cancer. Colorectal Dis. 2011;13(11): 1249-1255.

[31] Park JS, Chang IT, Park SJ, et al. Comparison of ex vivo and in vivo injection of blue dye in sentinel lymph node mapping for colorectal cancer. World J Surg. 2009;33(3):539-546.

[32] Andersen HS, Bennedsen ALB, Burgdorf SK, et al. In vivo and ex vivo sentinel node mapping does not identify the same lymph nodes in colon cancer. Int J Colorectal Dis. 2017;32(7):983-990.

[33] Weixler B, Rickenbacher A, Raptis DA, et al. Sentinel lymph node mapping with isosulfan blue or indocyanine green in colon cancer shows comparable results and identifies patients with decreased survival: a prospective single-center trial. World J Surg. 2017;41(9):2378-2386.

[34] Lezoche E, Fabiani B, D’Ambrosio G, et al. Nucleotide-guided mesorectal excision combined with endoluminal locoregional resection by transanal endoscopic microsurgery in the treatment of rectal tumors: technique and preliminary results. Surg Endosc. 2013;27(11):4136-4141.

[35] Arezzo A, Arolfo S, Mistrangelo $M$, et al. Transrectal sentinel lymph node biopsy for early rectal cancer during transanal endoscopic microsurgery. Minim Invasive Ther Allied Technol. 2014;23(1): 17-20. 